Suneela Vegunta, MD

Division of Women's Health Internal Medicine,

Mayo Clinic, Scottsdale, AZ
Margaret E. Long, MD

Department of Obstetrics and Gynecology,

Mayo Clinic, Rochester, MN

\title{
Q: Should women with HPV-related noncervical cancers be considered at high risk for cervical cancer?
}

\begin{abstract}
A: Yes. Human papillomavirus (HPV) infection causes cancer in more than just the cervix, and the incidence of high-grade cervical lesions is higher for women with noncervical HPV-related cancers. Currently, however, no guidelines exist for screening for cervical cancer in women who have other HPV-related cancers. Nevertheless, screening and early diagnosis reduce the risks of cancer-related comorbid conditions and death, so until clear guidelines are available, the current evidence suggests that these women should be considered at high risk and offered closer surveillance.
\end{abstract}

See related editorial, page 545

\section{HIGH-RISK HPV GENOTYPES}

HPV is the most common sexually transmitted infection in the United States, with greater than half the population infected at least once during their lifetime. ${ }^{1-3}$ Of the more than 100 known HPV genotypes, there are 15 high-risk oncogenic types: 16, 18, 31, $33,35,39,45,51,52,56,58,59,68,73$, and 82.

Persistent infection with high-risk HPV causes most cases of squamous cell carcinoma of the cervix, vulva, vagina, oropharynx, penis, and anal canal. ${ }^{4}$ This causation is supported by epidemiologic and molecular studies, and highrisk HPV DNA can be found in most of these carcinomas. Infection with low-risk genotypes (HPV-6 and HPV-11) can result in benign anogenital warts and respiratory papillomatosis but not cancer.

doi:10.3949/ccjm.87a.19145

\section{HPV-RELATED CANCERS}

Most HPV infections are transient and resolve or become dormant within 2 years, but a few women with persistent high-risk HPV infection develop cervical cancer. With improved screening, early diagnosis, and treatment of precancerous lesions, the incidence and mortality rates of cervical cancer have decreased, and these rates are expected to decrease further with adequate HPV vaccination., 5,6

The only HPV vaccine currently available in the United States protects against 9 HPV serotypes, including HPV-16 and HPV18 , which cause more than $60 \%$ of HPV cancer cases in men and women, including most cervical, anal, and oropharyngeal cancers. ${ }^{4}$ In particular, HPV-16 causes more cases of cancer than any other HPV serotype. Around the world, vaccination provides the opportunity to decrease the burden of high-risk HPV infections in future generations.

The HPV Vaccine Impact Monitoring Project (HPV-IMPACT) study ${ }^{7}$ collected data on cervical lesions attributable to HPV-16 and HVP-18 and also examined vaccination rates in women ages 18 to 39 . The vaccinated women had fewer lesions than those who were not vaccinated.

For men and women, the incidence rates of high-risk HPV at multiple anatomic sites are increasing. Without effective screening programs, the incidence of high-risk HPVmediated oropharyngeal, vulvar, and anal squamous cell carcinoma likewise is steadily increasing. Certain sexual behaviors (eg, unprotected oral sex, receptive anal sex) and other factors such as impaired local mucosal and systemic immunity increase the risk of

\section{Lacking guidelines, a prudent strategy is to increase screening in this group}

TO SPECIFIC

CLINICAL QUESTIONS 
HPV infection and subsequent cancer.

Anal cancer is slightly more common in women than in men. However, the rate of anal cancer is especially high in men positive for human immunodeficiency virus (HIV) who have sex with men, and it is elevated to a lesser extent in immunosuppressed individuals. ${ }^{8,9}$ The anus and cervix, which share embryologic and anatomic characteristics, may respond similarly to malignant changes induced by persistent high-risk HPV infection, and a clear majority of cancers at both these sites are attributable to HPV, ${ }^{10}$ as evidenced by detection in tumor specimens. ${ }^{11-13}$

In addition, according to the US Centers for Disease Control and Prevention, oropharyngeal squamous cell carcinoma is now the most common HPV-associated cancer, with incidence rates increasing by $2.7 \%$ per year in men and $0.8 \%$ per year in women. ${ }^{8}$ Oropharyngeal cancers are known to be caused by tobacco and alcohol use, but recent studies show that about $70 \%$ of these cancers are HPV-positive, with non-Hispanic White males having the highest risk.

\section{HPV AND CANCER SCREENING}

Cervical cancer

screening

guidelines

for HIV-positive

women

also apply to

immunosup-

pressed

women

without HIV

\section{Cervical cancer screening for high-risk groups}

Cervical cancer screening guidelines for immunocompetent females are based on data from 1.5 million women. ${ }^{14}$ However, with their increased risk of cervical cancer, HIVpositive women have different screening guidelines based on HIV-specific data. Other women with suppressed immune function or immune dysfunction due to solid-organ transplant, bone marrow transplant, or diseasemodifying therapy for autoimmune disease, as well as women with inflammatory bowel disease who are receiving immunosuppressive therapy, also have increased risk of high-grade squamous intraepithelial lesions of the cervix and squamous cell carcinoma. ${ }^{15}$

Primarily based on expert opinion but also on available data, cervical cancer screening guidelines for HIV-positive women (noted below) also apply to immunosuppressed women without HIV:

- Cotesting (HPV and cervical cytology) every 3 years, if preferred, for women 30 years of age and older.

- If HPV testing is unavailable or if the woman is age 21 to 29 , cervical cytology is indicated annually for 3 years, and then once every 3 years thereafter if initial results are negative.

- Screening continues (past age 65) as long as the patient's health supports continued screening.

Women who were exposed to diethylstilbestrol in utero have an increased risk of clear-cell adenocarcinoma of the cervix, a rare cancer unrelated to HPV. For these women, annual cervical cytologic examination is currently recommended. ${ }^{16}$

\section{Screening women with lower genital tract cancers for anal dysplasia, cancer}

The progression from HPV acquisition to persistent HPV infection to precancerous and cancerous cervical lesions is well established. Other noncervical HPV-related cancers have not been studied as extensively, but an event progression similar to that of cervical cancer has been hypothesized.

Patients with HPV infection at a single anatomic site have a higher risk of infection at other HPV-related sites through autoinfection. ${ }^{17}$ For some women with concurrent cervical and anal high-risk HPV infections, the genotypes have shown a high degree of concordance. High-risk HPV infection and coexisting abnormal anal and cervical cytologic findings are common in women receiving immunosuppressive therapy. ${ }^{18}$ Women who have been treated for high-risk HPV-positive anal cancer have high rates of persistent anal HPV infection, ${ }^{19}$ which can lead to infection of other genital sites. ${ }^{20}$

Likewise, the prevalence of anal intraepithelial lesions is increased in women with HPV-related high-grade squamous intraepithelial cervical lesions, ${ }^{21}$ and this prevalence is greater in women with cervical cancer and greater still in women with HPV-mediated vulvar cancer. ${ }^{17}$ Cervical, vulvar, vaginal, and anal dysplasia and cancers can be considered parts of a multicentric disease of the lower anogenital tract.

Women with cervical, vulvar, and vaginal cancer have a higher risk of anal cancer. Screening recommendations for anal cancer 
in these women, at a minimum, should include annual symptom query, visual inspection, and digital anorectal examination. Anal cytology can be considered, depending on the degree of anal cancer risk and the availability of colorectal surgeons who can perform the high-resolution anoscopy required after abnormal cytologic results. ${ }^{21}$ The annual anal cytology that some recommend for women with HIV ${ }^{22}$ could benefit women with highgrade vulvar dysplasia and cancer because their anal cancer rates are similar. ${ }^{1,10}$ Limited data on the natural course of anal dysplasia in women with HPV-mediated cancers hinders the creation of evidence-based guidelines.

\section{Cervical cancer screening for women with noncervical HPV-related cancers}

Evidence suggests that the risk of cervical cancer may increase for women with other HPVrelated cancers, but currently, we lack specific data and guidance about screening for cervical cancer in women with other HPV-related cancers. This area of ambiguity needs further research. Until then, on the basis of currently available evidence, these women should be considered at high risk and offered cervical cancer screening similar to that of women with HIV infection, as previously detailed.

Because most cases of cervical cancer in the United States are in women who are unscreened or underscreened, we recommend reviewing results of any prior cervical screening. Cervical HPV testing with genotyping and reflex cytologic testing (ie, primary HPV screening) or HPV and cytologic testing (ie, cotesting) should be performed if these tests are available and prior results are not current or cannot be reviewed. Negative HPV test results provide strong evidence of reduced risk of cervical cancer.

Prevention and treatment of precancerous cervical lesions reduce the rate of cervical cancer. In one study, treatment of cervical intraepi-

\section{TABLE 1}

\section{Suggested screening guidelines}

\section{Cervical cancer screening for women with other HPV-related cancer}

Cotesting HPV and cervical cytology every 3 years; preferred for women age 30 and older

Annual cervical cytology for 3 years, then every 3 years if negative; preferred for women under age 30 , or if HPV testing is unavailable

Anal cancer screening for women with other HPV-related high-grade anogenital dysplasia or cancer

Annual symptom query, visual inspection, and digital anorectal examination

Anal cytology can be considered, depending on the availability of high-resolution anoscopy (high-grade vulvar dysplasia and cancer are associated with higher risk than at other sites)

thelial neoplasia grade 3 reduced cancer risk from $30 \%$ to $1 \%{ }^{23}$ Thus, the recommended follow-up for abnormal cervical cancer screening results must occur in a timely manner.

\section{A PRUDENT STRATEGY}

The global burden of HPV-related cancers is increasing, but the incidence of these cancers can be reduced by broadly increasing HPV vaccination rates for both sexes worldwide. Because HPV is a multicentric disease, women with lower genital tract cancers should be considered to have higher risk for anal cancers, and women with noncervical HPVrelated cancers should be considered to have a higher risk for cervical cancer.

A prudent strategy would be to offer these women closer surveillance, and the suggested screening guidelines are summarized in Table 1. More research is needed to provide clear guidelines for cervical cancer screening for women with other HPV-related cancers.

\section{REFERENCES}

1. Moscicki AB, Darragh TM, Berry-Lawhorn JM, et al. Screening for anal cancer in women. J Low Genit Tract Dis 2015; 19(3 suppl 1):S27S42. doi:10.1097/LGT.0000000000000117

2. Myers ER, McCrory DC, Nanda K, Bastian L, Matchar DB. Mathematical model for the natural history of human papillomavirus infection and cervical carcinogenesis. Am J Epidemiol 2000; 151(12):11581171. doi:10.1093/oxfordjournals.aje.a010166

3. Beachler DC, Kreimer AR, Schiffman M, et al. Multisite HPV16/18 vaccine efficacy against cervical, anal, and oral HPV infection. J Natl Cancer Inst 2015; 108(1):djv302. doi:10.1093/jnci/djv302

4. Gillison ML, Chaturvedi AK, Anderson WF, Fakhry C. Epidemiology of human papillomavirus-positive head and neck squamous cell carcinoma. J Clin Oncol 2015; 33(29):3235-3242. doi:10.1200/JCO.2015.61.6995

5. National Cancer Institute. SEER cancer statistics review (CSR) 1975-2015 https://seer.cancer.gov/archive/csr/1975_2015/. Accessed August 5, 2020.

6. Watson M, Soman A, Flagg EW, et al. Surveillance of high-grade cervical cancer precursors (CIN III/AIS) in four population-based can- 
cer registries, United States, 2009-2012. Prev Med 2017; 103:60-65. doi:10.1016/j.ypmed.2017.07.027

7. Hariri S, Bennett NM, Niccolai LM, et al. Reduction in HPV 16/18-associated high grade cervical lesions following HPV vaccine introduction in the United States-2008-2012. Vaccine 2015; 33(13):16081613. doi:10.1016/j.vaccine.2015.01.084

8. Van Dyne EA, Henley SJ, Saraiya M, Thomas CC, Markowitz LE, Benard VB. Trends in human papillomavirus-associated cancersUnited States, 1999-2015. MMWR Morb Mortal Wkly Rep 2018; 67(33):918-924. doi:10.15585/mmwr.mm6733a2

9. National Cancer Institute. Cancer stat facts: anal cancer. https://seer. cancer.gov/statfacts/html/anus.html. Accessed August 5, 2020.

10. Saleem AM, Paulus JK, Shapter AP, Baxter NN, Roberts PL, Ricciardi R. Risk of anal cancer in a cohort with human papillomavirus-related gynecologic neoplasm. Obstet Gynecol 2011; 117(3):643-649. doi:10.1097/AOG.0b013e31820bfb16

11. Hoots BE, Palefsky JM, Pimenta JM, Smith JS. Human papillomavirus type distribution in anal cancer and anal intraepithelial lesions. Int J Cancer 2009; 124(10):2375-2383. doi:10.1002/ijc.24215

12. Alemany L, Saunier M, Alvarado-Cabrero I, et al. Human papillomavirus DNA prevalence and type distribution in anal carcinomas worldwide. Int J Cancer 2015; 136(1):98-107. doi:10.1002/ijc.28963

13. Morel A, Neuzillet C, Wack M, et al. Mechanistic signatures of human papillomavirus insertions in anal squamous cell carcinomas. Cancers (Basel) 2019; 11(12):1846. doi:10.3390/cancers11121846

14. Perkins RB, Guido RS, Castle PE, et al. 2019 ASCCP risk-based management consensus guidelines for abnormal cervical cancer screening tests and cancer precursors. J Low Genit Tract Dis 2020; 24(2):102-131. doi:10.1097/LGT.0000000000000525

15. Moscicki $A B$, Flowers $L$, Huchko MJ, et al. Guidelines for cervical cancer screening in immunosuppressed women without HIV infection. J Low Genit Tract Dis 2019; 23(2):87-101. doi:10.1097/LGT.0000000000000468

16. National Cancer Institute. Diethylstilbestrol (DES) and cancer. https:// www.cancer.gov/about-cancer/causes-prevention/risk/hormones/desfact-sheet. Accessed August 5, 2020.

17. Fokom Domgue J, Messick C, Milbourne A, et al. Prevalence of highgrade anal dysplasia among women with high-grade lower genital tract dysplasia or cancer: results of a pilot study. Gynecol Oncol 2019; 153(2):266-270. doi:10.1016/j.ygyno.2019.02.024

18. Eleutério J Jr, Cavalcante LR, Gonçalves AKS, Eleutério RMN, Giraldo PC. Prevalence of high-risk HPV and atypia in liquid-based cytology of cervical and intra-anal specimens from kidney-transplanted women. Diagn Cytopathol 2019; 47(8):783-787. doi:10.1002/dc.24180

19. Chin-Hong PV, Palefsky JM. Natural history and clinical management of anal human papillomavirus disease in men and women infected with human immunodeficiency virus. Clin Infect Dis 2002; 35(9):1127-1134. doi:10.1086/344057

20. Parkin DM, Bray F. Chapter 2: the burden of HPV-related cancers. Vaccine 2006; 24(suppl 3):S3/11-S3/25. doi:10.1016/j.vaccine.2006.05.111

21. Hillman RJ, Berry-Lawhorn JM, Ong JJ, et al. International Anal Neoplasia Society guidelines for the practice of digital anal rectal examination. J Low Genit Tract Dis 2019; 23(2):138-146. doi:10.1097/LGT.0000000000000458

22. National AIDS Treatment Advocacy Program. NYS guidelines recommendations on anal Pap smears. http://www.natap.org/2010/ HIV/032510_01.htm. Accessed August 5, 2020.

23. Lili E, Chatzistamatiou K, Kalpaktsidou-Vakiani A, Moysiadis T, Agorastos $\mathrm{T}$. Low recurrence rate of high-grade cervical intraepithelial neoplasia after successful excision and routine colposcopy during follow-up. Medicine (Baltimore) 2018; 97(4):e9719. doi:10.1097/MD.0000000000009719

Address: Suneela Vegunta, MD, Division of Women's Health Internal Medicine, Mayo Clinic, 13400 E Shea Boulevard, Scottsdale, AZ 85259; vegunta.suneela@mayo.edu 